\title{
Engineering transverse Bragg resonance waveguides for large modal volume lasers
}

\author{
Wei Liang, Yong Xu, John M. Choi, and Amnon Yariv \\ Department of Applied Physics, California Institute of Technology, Pasadena, California 91125
}

Received May 8, 2003

\begin{abstract}
We recently analyzed a new class of laser amplifier based on transverse Bragg reflection. We show that the unique properties of Bragg confinement make it possible through modal loss discrimination to achieve single-transverse-mode operation with transverse modal size that is an order of magnitude larger than in lasers that depend on total internal reflection for transverse confinement. () 2003 Optical Society of America

OCIS codes: 130.2790, 140.3280, 140.3490, 250.4480.
\end{abstract}

It is well known that one can form one-dimensional photonic crystal waveguides, i.e., Bragg waveguides, by Bragg reflection from periodic cladding rather than from total internal reflection. ${ }^{1}$ Recently a new type of two-dimensional photonic crystal waveguide, the transverse Bragg resonance (TBR) waveguide, was proposed and analyzed. ${ }^{2,3}$ A unique feature of the TBR structures is the large modal cross section, which makes them particularly attractive for applications such as high-power delivery and high-power lasers, for which the maximal power is limited by the transverse modal size and the power density at catastrophic optical mirror damage of the end facets. ${ }^{4,5}$ In conventional waveguides based on total internal reflection, to achieve good beam quality (single-lateral-mode operation) the core width is typically limited to several micrometers, such as in the well-known ridge waveguide laser. ${ }^{5}$ In this Letter we investigate the condition of single-transverse-mode guiding in a TBR waveguide with a much larger core width and demonstrate that the TBR waveguide can possess a much larger core width and thus can have a significant advantage over the traditional total internal reflection waveguides for high-power laser applications. Futhermore, the basic idea proposed here can be used to increase the core widths of other types of photonic crystal waveguides.

As shown in Fig. 1(a), the TBR waveguide consists of a slab (with dielectric constant $\varepsilon_{\text {co }}$ and width $W_{\text {co }}$ ) flanked by two Bragg reflectors, which alternate between a high-index layer with dielectric constant $\varepsilon_{1}$ and layer thickness $L_{1}$ and a low-index layer with dielectric constant $\varepsilon_{2}$ and layer thickness $L_{2}$. In this Letter we consider only small index contrast, with $\varepsilon_{\mathrm{co}}=\varepsilon_{2} \cong \varepsilon_{1}$. We further require that $L_{1}=L_{2}=b / 2$, which provides optimal confinement according to the results in Refs. 2 and 3. The $z$ dependence of the guided mode can be described by a complex propagation constant $\beta=\beta_{R}+i \beta_{I}$ by the factor $\exp (-i \beta z)$. Using the boundary condition of no incoming wave at the cladding edge, we find that a guided mode in a passive TBR waveguide satisfies the following phase condition $^{2,3}$ :

$$
\begin{aligned}
k_{\mathrm{co}} W_{\mathrm{co}} & =m \pi+\text { phase }\left[\frac{\kappa}{\gamma-i \Delta k-S \operatorname{coth}(S L)}\right], \\
m & = \begin{cases}\text { even } & \text { even mode } \\
\text { odd } & \text { odd mode }\end{cases}
\end{aligned}
$$

where $m$ is an integer, $S=\left[|\kappa|^{2}+(\gamma-i \Delta k)^{2}\right]^{1 / 2}$, $\gamma=-\beta_{R} \beta_{1} / k_{0}, \quad k_{0}^{2}=(\omega / c)^{2}\left(\varepsilon_{1}+\varepsilon_{2}\right) / 2-\beta_{R}{ }^{2}$, $k_{\mathrm{co}}{ }^{2}=(\omega / c)^{2} \varepsilon_{\mathrm{co}}-\beta_{R}{ }^{2}, \Delta k=k_{0}-\pi / b$, and $\kappa=\omega^{2}\left(\varepsilon_{1}-\varepsilon_{2}\right) /\left(2 k_{0} c^{2} \pi\right) . \quad \kappa$ is the coupling constant
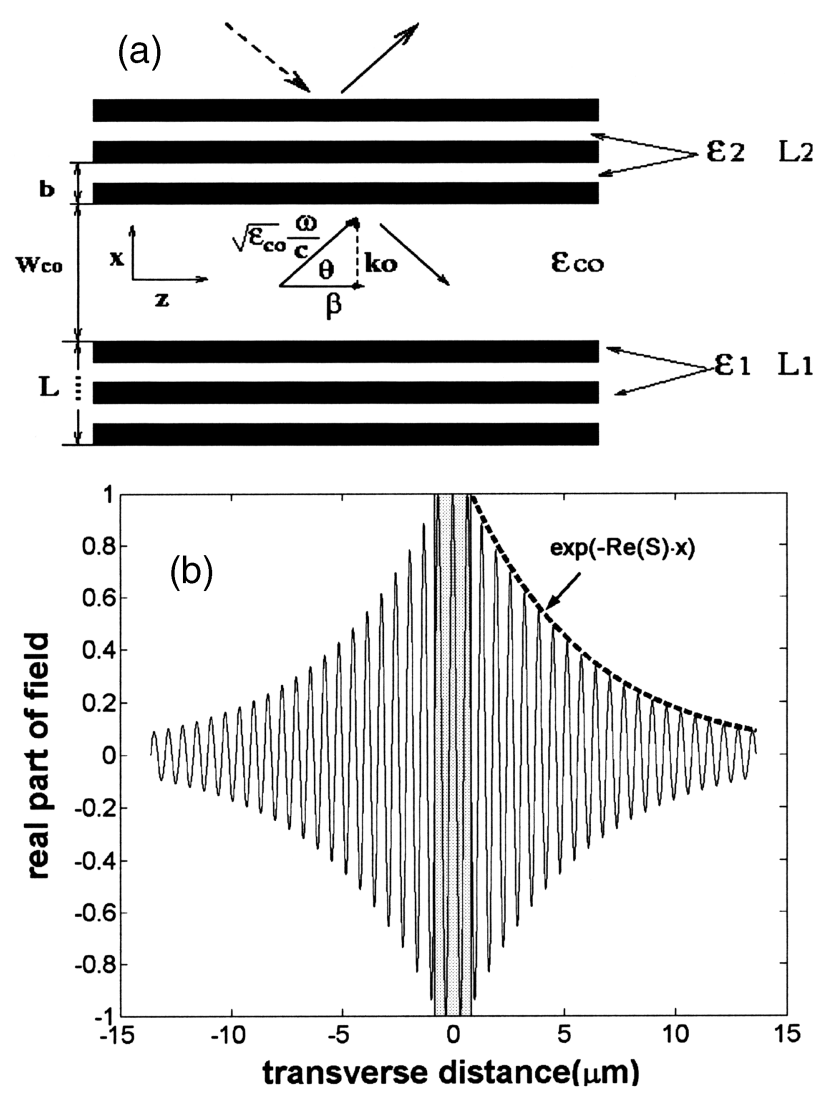

Fig. 1. (a) Schematic of a TBR waveguide. (b) Transverse field distribution of a guided TBR mode. Shaded central region, waveguide core; dashed curves, exponential decay of the form $\exp [-\operatorname{Re}(S)|x|]$. 
of the Bragg reflector and $k_{0}$ and $k_{\text {co }}$, respectively, represent the transverse wave vector in the cladding and the core regions. For small index contrast $\varepsilon_{\text {co }}=\varepsilon_{2} \cong \varepsilon_{1}$, we have $k_{0} \cong k_{\text {co }}$. Consider the field profile obtained in Ref. 3 for $\operatorname{Re}(S L) \gg 1$. The envelope of the cladding field can be well approximated by an exponential decay $\exp [-\operatorname{Re}(S)|x|]$. The transverse field profile of a guided mode calculated with the coupled-mode formalism is plotted in Fig. 1(b). An envelope function of the form $\exp [-\operatorname{Re}(S)|x|]$ is given in Fig. 1(b) by the dashed curve, which shows a good match with the coupled-mode results. For the calculations we chose typical parameters $\lambda=1.55 \mu \mathrm{m}$, $\varepsilon_{1}=12.96$, and $b=0.32 \mu \mathrm{m}$, which are used throughout the rest of the Letter. The additional parameters are $\varepsilon_{\text {co }}=\varepsilon_{2}=12.25, W_{\text {со }}=1.65 \mu \mathrm{m}$, and $L=12.8 \mu \mathrm{m}$.

For integrated optics applications, the waveguide loss is typically limited to a level of less than $1 \mathrm{~dB} / \mathrm{cm}$, which corresponds to $\gamma \sim \beta_{\mathrm{I}} \approx 10^{-5} \mu \mathrm{m}^{-1}$, whereas, according to the definition following Eq. (1), $|\kappa|$ is approximately $10^{-2} \mu \mathrm{m}^{-1}$ for $\varepsilon_{1}-\varepsilon_{2} \sim 10^{-1}$. As a result, we can ignore $\gamma$ and put $S=\left(|\kappa|^{2}-\Delta k^{2}\right)^{1 / 2}$. In Fig. 2(a) we use $\gamma=\beta_{\mathrm{I}}=0$ and show the real part of $S$ as a function of $\beta_{R} c / \omega$ for a TBR waveguide with $\varepsilon_{\mathrm{co}}=\varepsilon_{2}=12.25$. A confined mode requires a decaying cladding field and occurs only if $\operatorname{Re}(S)>0$ and consequentially $\Delta k<|\kappa|$. This in turn limits the possible values of the real part of propagation constant $\beta_{R} c / \omega$ of the guided modes within the bandgap region, as shown in Fig. 2(a). A well-confined TBR mode also requires that $\operatorname{Re}(S) L \gg 1$. We can simplify Eq. (1) under these approximations into

$$
\begin{aligned}
k_{\mathrm{co}} W_{\text {со }} & =m \pi+\Phi, \\
\Phi & =\text { phase }\left\{\kappa /\left[-i \Delta k-\left(|\kappa|^{2}-\Delta k^{2}\right)^{1 / 2}\right]\right\} .
\end{aligned}
$$

Considering a multimode (same-frequency) TBR waveguide with a large core area; according to Eq. (2), two neighboring modes should satisfy $k_{\mathrm{co}, 1} W_{\mathrm{co}}=m \pi+\Phi_{1}$, $k_{\mathrm{co}, 2} W_{\mathrm{co}}=(m+1) \pi+\Phi_{2}$. The difference between the two transverse wave vectors is $\Delta k_{\text {diff }}=k_{0,2}-k_{0,1}=$ $\left(\pi+\Phi_{2}-\Phi_{1}\right) / W_{\mathrm{co}}, k_{0} \cong k_{\mathrm{co}}$. If $\Delta k_{\text {diff }}$ is smaller than $|\kappa|$, multiple transverse modes occur. For example, in Fig. 2(b) we show the appearance of multiple guided modes for a large-core-area TBR waveguide with $W_{\text {co }}=$ $49.85 \mu \mathrm{m}$ and $\varepsilon_{\mathrm{co}}=\varepsilon_{2}=12.25$.

Using Eq. (2), we can estimate the number of transverse modes in a large-core-area TBR waveguide. As the total change of $\Phi$ is $\pi$ within the bandgap region $|\Delta k|<|\kappa|$, we treat it is a slowly varying variable and ignore the term $\Phi_{2}-\Phi_{1}$ in the expression for $\Delta k_{\text {diff }}$. Under this assumption, for a given bandgap region $|\Delta k|<|\kappa|$ the total number of guided modes is

$$
N_{\text {mod e }} \cong \frac{2|\kappa|}{\Delta k_{\text {diff }}} \cong \frac{2|\kappa|}{\pi / W_{\text {co }}}=\frac{2|\kappa|}{\pi} W_{\text {co }} .
$$

Applying Eq. (3) to the TBR waveguide studied in Fig. 2(b), we obtain an estimate of $N_{\text {mode }}=6$, which is a reasonable value compared with the five guided modes shown in Fig. 2(b).

From Eq. (3) we can define $W_{\text {co }}{ }^{\mathrm{sig}}=\pi / 2|\kappa|$ as the largest core width of a TBR waveguide that can support only one mode. In Table 1 we list the estimation of $W_{\text {co }}{ }^{\text {sig }}$ for three values of dielectric constant contrast in the TBR cladding. In a TBR waveguide the guided modes penetrate substantially into the cladding structures [as can be seen from Fig. 1(b)], with a penetration depth of $1 / \operatorname{Re}(S)$. Therefore, for these single-mode TBR waveguides, we can define the effective modal width as $W_{\text {co }}{ }^{\text {sig }}+2 / \operatorname{Re}(S)$. For $\Delta \varepsilon=0.072$ in Table 1 (corresponding to an index contrast of $\Delta n=0.02$ ), the effective modal width approaches $93 \mu \mathrm{m}$, which is $\sim 20$ times larger than that of the state-of-the art single-ridge waveguide high-power semiconductor laser. ${ }^{5}$

For applications such as high-power lasers it is possible to use $W_{\text {co }}$ larger than $W_{\text {co }}{ }^{\text {sig }}$ and still maintain single-transverse-mode operation. The key reason is that multiple transverse modes have significantly different propagation loss, a unique feature to the TBR waveguide. For example, in Fig. 2(b) the propagation loss of the centered mode is $0.0154 \mathrm{~dB} / \mathrm{cm}$, which is smaller than 0.0242 and $0.0235 \mathrm{~dB} / \mathrm{cm}$, the losses of the two side modes, respectively. This modal loss discrimination can be attributed to the fact that different transverse modes occupy different positions within the bandgap region, with maximal confinement (least propagation loss) achieved at the band center $(\Delta k=0, S=|\kappa|)$.

To quantify the degree of single-mode lasing in a multimode TBR waveguide we use the concept of the mode suppression ratio (MSR), which is defined as the ratio of the output power in the main lasing mode to that in the next-strongest side mode and can be estimated $a^{6}$

$$
\mathrm{MSR} \approx \frac{\Delta \alpha+\Delta g}{\delta_{G}}+1
$$

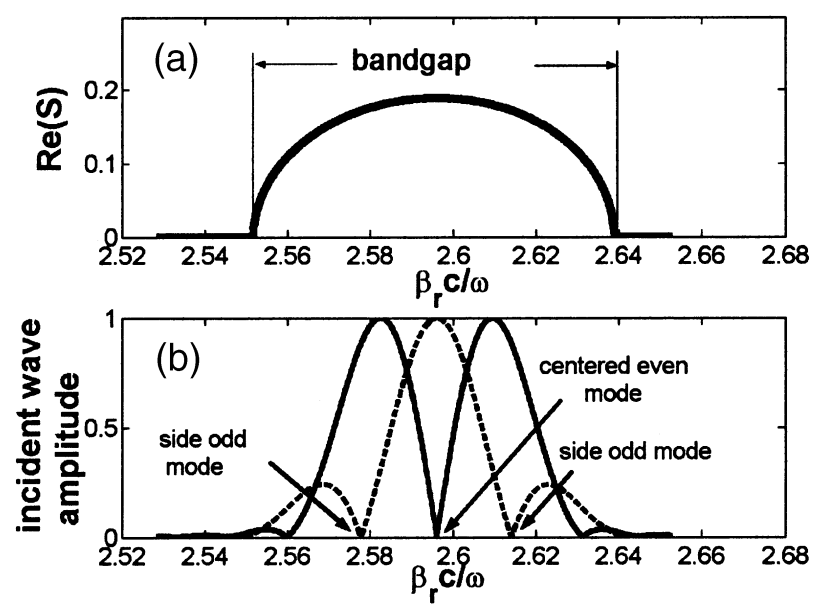

Fig. 2. (a) $\operatorname{Re}(S)$ as a function of effective index $\beta_{r} c / \omega$. A confined mode in a TBR waveguide is possible only in the bandgap region, with $\operatorname{Re}(S)>0$. (b) Amplitude of the incident wave [dashed arrow in Fig. 1(a)] as a function of effective index. Solid curve, solutions with even symmetry; dashed curve, solutions of odd symmetry. Within the bandgap, at each zero-value point, Eq. (1) is satisfied, which indicates the existence of a guided TBR mode. Here we use $\beta_{i}=0, \varepsilon_{\text {co }}=\varepsilon_{2}=12.25, W_{\text {co }}=49.85 \mu \mathrm{m}$, and $L=32 \mu \mathrm{m}$. 
Table 1. Parameters for Quasi-Single-Mode Guiding in a TBR Waveguide ${ }^{a}$

\begin{tabular}{|c|c|c|c|c|c|c|}
\hline$\Delta \varepsilon$ & $1 / \kappa(\mu \mathrm{m})$ & $W_{\text {co }} \operatorname{sig}(\mu \mathrm{m})$ & $L(\mu \mathrm{m})$ & $W_{\text {со }}{ }^{a}(\mu \mathrm{m})$ & $W_{\text {со }}^{b}(\mu \mathrm{m})$ & $W_{\mathrm{co}}^{c}(\mu \mathrm{m})$ \\
\hline 0.710 & 5.29 & 8.31 & 19.2 & 20.14 & 36.65 & 82.87 \\
\hline 0.358 & 10.50 & 16.50 & 35.2 & 36.07 & 75.07 & 162.82 \\
\hline 0.144 & 26.04 & 40.90 & 96 & 64.72 & 122.67 & 254.68 \\
\hline
\end{tabular}

${ }^{a} W_{\text {co }}$ sig is the largest core width that satisfies the condition of single-mode guiding. The last three columns $W_{\text {co }}{ }^{2}, W_{\text {co }}{ }^{b}$, and $W_{\mathrm{co}}{ }^{c}$, satisfy the condition of quasi-single mode guiding and correspond, respectively, to approximate MSR values of $\sim 10,000, \sim 1000$, and $\sim 100$.

where $\Delta \alpha$ and $\Delta g$ are, respectively, the loss and gain differences between the main mode and the side mode and $\delta_{G}$ is the difference between the total loss and the modal gain for the main lasing mode. From a rate equation analysis it can be shown that the modal gain of the main lasing mode is always slightly smaller than the cavity loss at steady-state operation and that $\delta_{G}$ has a typical value of $\sim 10^{-4} \mathrm{~cm}^{-1}$ for an injection current that is ten times above the threshold value. ${ }^{7}$ In this Letter we consider only multiple transverse modes with the same frequency and assume that the main mode and the side modes correspond, respectively, to the centered mode with the lowest propagation loss and the side modes with the second-lowest propagation loss shown in Fig. 2(b). Because the different modes have the same frequency and similar field distribution, gain difference $\Delta g$ is quite small, and the main contribution to the MSR comes from $\Delta \alpha$. If the mirror loss difference is ignored, $\Delta \alpha$ is simply the propagation loss difference between the main mode and the side mode. We chose the width of the Bragg cladding, $L$, to keep the loss of the main mode at the level of $1 \mathrm{~dB} / \mathrm{cm}$. In the three rightmost columns of Table 1 we give core widths $W_{\text {co }}$ that correspond to MSRs of $\sim 10,000, \sim 1000$, and $\sim 100$, respectively. We observe that for $\Delta \varepsilon=0.144$ the TBR laser can maintain single-transverse-mode operation with a core width as large as $255 \mu \mathrm{m}$. As a result, we expect that the TBR lasers will lead to a significant boost of the output power of high-power lasers.

There are several nonlinear mechanisms (carrierinduced index change is the dominant one) that might degrade the single-lateral-mode operation of a TBR semiconductor laser. ${ }^{8}$ The carrier-induced index change can be estimated from $\Delta n \approx n_{2} I$, where $n_{2}$ is typically $\sim 2 \times 10^{-10} \mathrm{~cm}^{2} / \mathrm{W}{ }^{8}$ If we use an optical power density $I$ that corresponds to the threshold for mirror damage, which is approximately $10 \mathrm{MW} / \mathrm{cm}^{2}$ for cw operation, ${ }^{4}$ we find that the maximum carrier-induced index change is $\Delta n \approx 0.002$, which corresponds to one tenth of the smallest index contrast used in Table 1 . In this case, if we assume a worst-case situation and assume that the index contrast is changed to $\Delta n=0.022$ as a result of the carrier-induced index change, calculations show that the MSR will be reduced by a factor of 2 , which is more than enough for achieving single-lateral-mode operation. Therefore we believe that the carrierinduced index change should not significantly degrade the single-transverse-mode operation of a high-power semiconductor TBR laser.

This research was funded by the U.S. Office of Naval Research (Y. S. Park), whose support is gratefully acknowledged. J. Choi thanks the American Society for Engineering Education and the U.S. Department of Defense for their support. W. Liang's e-mail address is liangwei@its.caltech.edu.

\section{References}

1. P. Yeh and A. Yariv, Opt. Commun. 19, 427 (1976).

2. A. Yariv, Opt. Lett. 27, 936 (2002).

3. A. Yariv, Y. Xu, and S. Mookherjea, Opt. Lett. 28, 176 (2003).

4. D. Botez, Appl. Phys. Lett. 74, 3102 (1999).

5. G. W. Yang, R. J. Hwu, Z. T. Xu, and X. Y. Ma, IEEE J. Sel. Top. Quantum Electron. 6, 577 (2000).

6. L. A. Coldren and S. W. Corzine, in Diode Lasers and Photonic Integrated Circuits, K. Chang eds. (Wiley, New York, 1995), pp. 106-108.

7. C. L. Felix, I. Vurgaftman, W. W. Bewley, R. E. Bartolo, J. R. Lindle, J. R. Meyer, H. Lee, and R. U. Martinelli, J. Mod. Opt. 49, 801 (2002).

8. J. R. Marciante and G. P. Agrawal, IEEE J. Quantum Electron. 32, 590 (1996). 